\section{Gastric Cancer and Prediction of Prognosis}

\section{DEAR EDITORS:}

I am pleased to respond to the letter by Liakakos and Karatzas that highlights the limitation of predicting prognosis of gastric cancer based on metastatic lymph nodes number. ${ }^{1}$ Of note there are a number of factors aside from the number of metastatic lymph nodes that influence the prognosis of gastric cancer, such as depth of tumor invasion, tumor size, and Borrmann type, etc. The inclusion of other clinicopathological factors in the consideration of prognosis might be important. Indeed, there is a limitation of predicting prognosis of gastric cancer based on conventional clinicopathologic features, or International Union against Cancer (UICC) tumor-node-metastasis (TNM) stage. With the development of an understanding of the molecular mechanisms in gastric tumors, a more promising prognosis system based on genomics may be possible.

\section{Baojun Huang, MD}

Department of Surgical Oncology, First Affiliated Hospital of China Medical University, Liaoning, China

e-mail: huang_bj@163.com

Published Online: 29 January 2009

(C) Society of Surgical Oncology 2009

\section{REFERENCE}

1. Liakakos T, Karatzas G. Predicting prognosis of gastric cancer: limitations of metastatic lymph nodes number and genomics-based promises. Ann Surg Oncol. 2009. doi:10.1245/s10434-008-0305-4. 